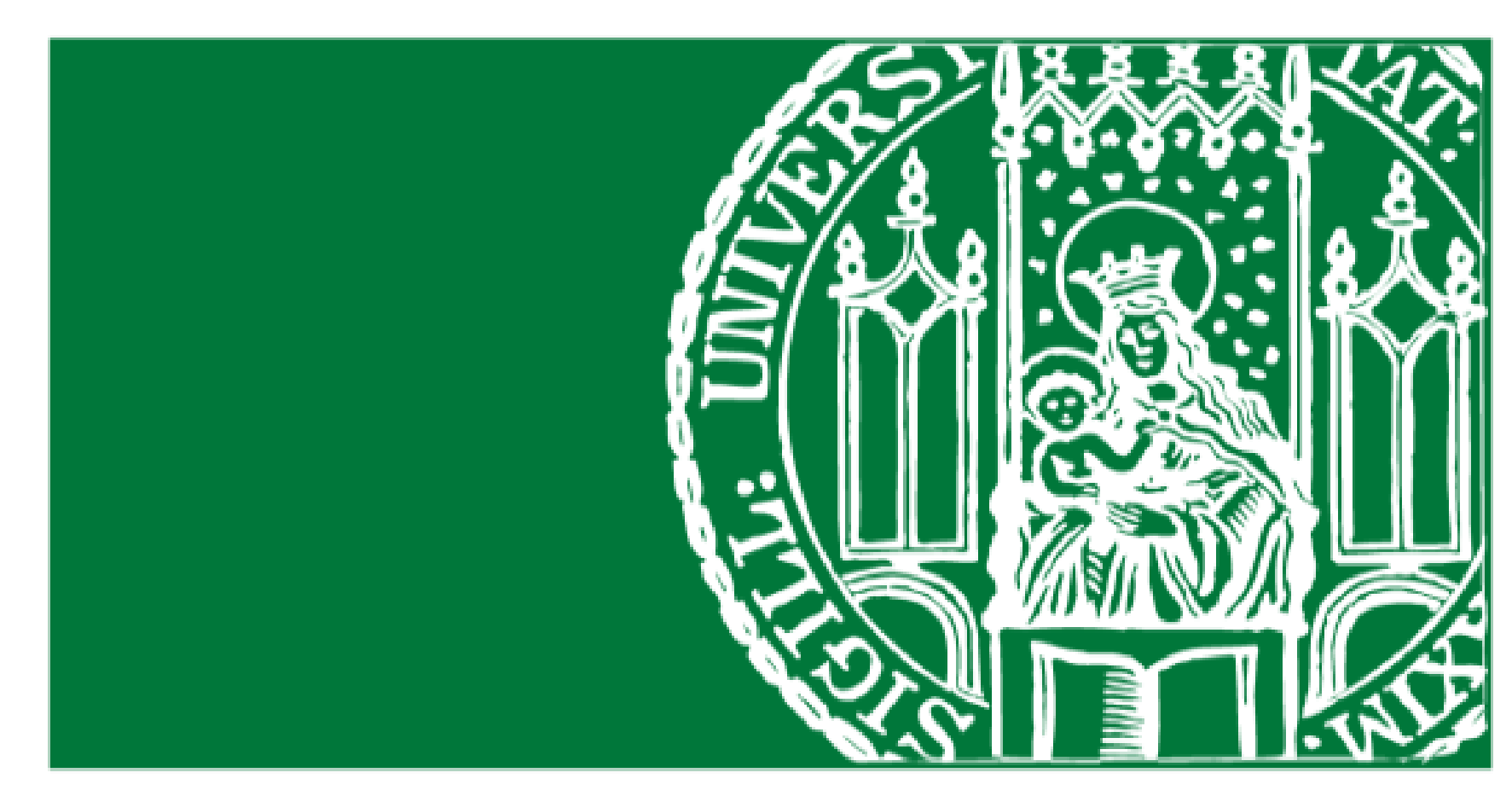

\title{
Synergistic anti-tumour effects of 13-cis retinoic acid and lovastatin in pancreatic neuroendocrine tumour (BON1) cells through enhanced EGFR inhibition \\ Svenja Nölting, Elke Tatjana Aristizabal Prada, Michael Lauseker, Julian Maurer, Gerald Spöttl, Burkhard Göke, Karel Pacak, Ashley Grossman
} Fig.3

Introduction

In our previous studies we found that the combination of 13-cis retinoic acid (13cRA) and lovastatin significantly reduced tumour growth in a mouse phaeochromocytoma allograft model, with the lowest microvessel density in the combination-treated tumours Nölting et al., Endocrinology, $2014 \mathrm{Jul}$ ). We have now investigated the effect of 13cRA plus lovastatin on neuroendocrine (BON1, H727) and nonendocrine tumour (HepG2, Huh7) cell viability and signalling pathways (EGFR, AKT, ERK, p70S6K) to elucidate the underlying mechanism of action.

Fig.1
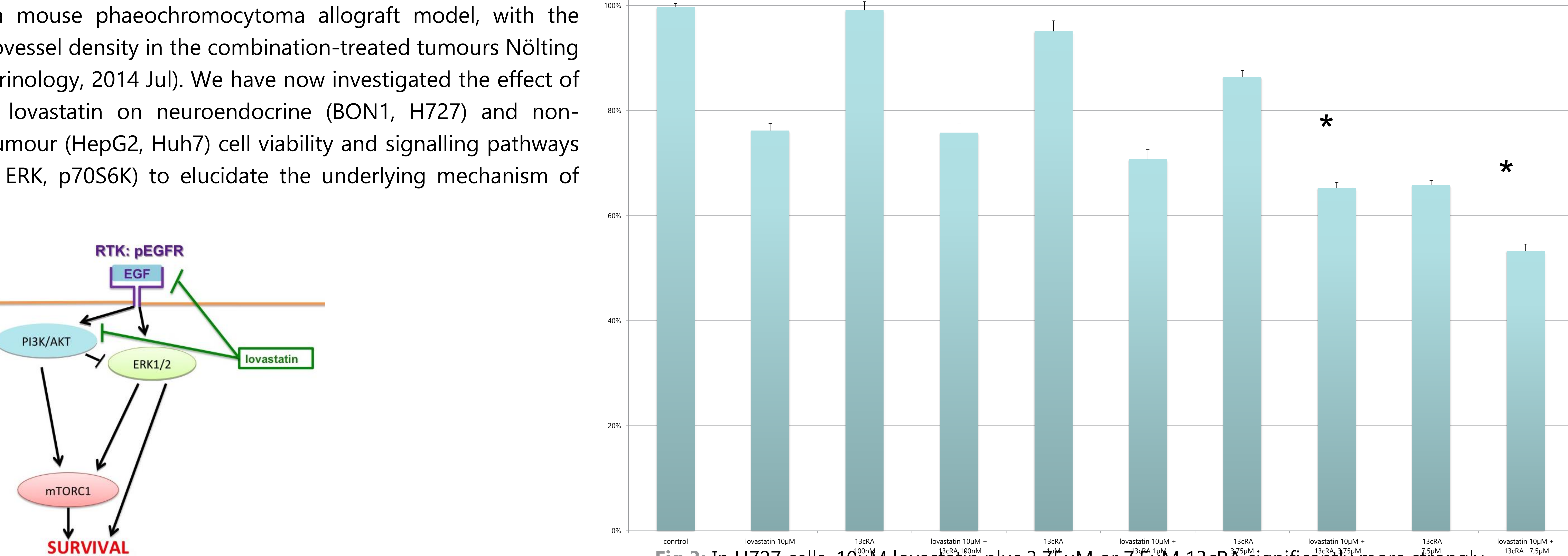

Fig.1: Effects of lovastatin on neuroendocrine tumour cells (modified from Nölting et al, PLOSone, 2015, Dec)

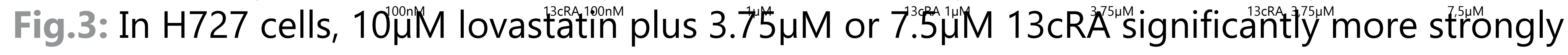
reduces cell viability than each drug separately $(p \leq 0.05)\left({ }^{*}\right)$, but this effect is not synergistic.

\section{Methods}

Cell viability was assessed with the MTS assay, the effect on signalling pathways by Western blotting. For cell viability data, the multiplecomparison Kruskal-Wallis-Test was used, followed by pairwise comparisons with the Mann-Whitney-Test. Interaction effects were analysed with LinearMixed-Effects-Models. Statistical significance was defined at $p \leq 0.05$.

Results

Fig.2

\section{BON1}
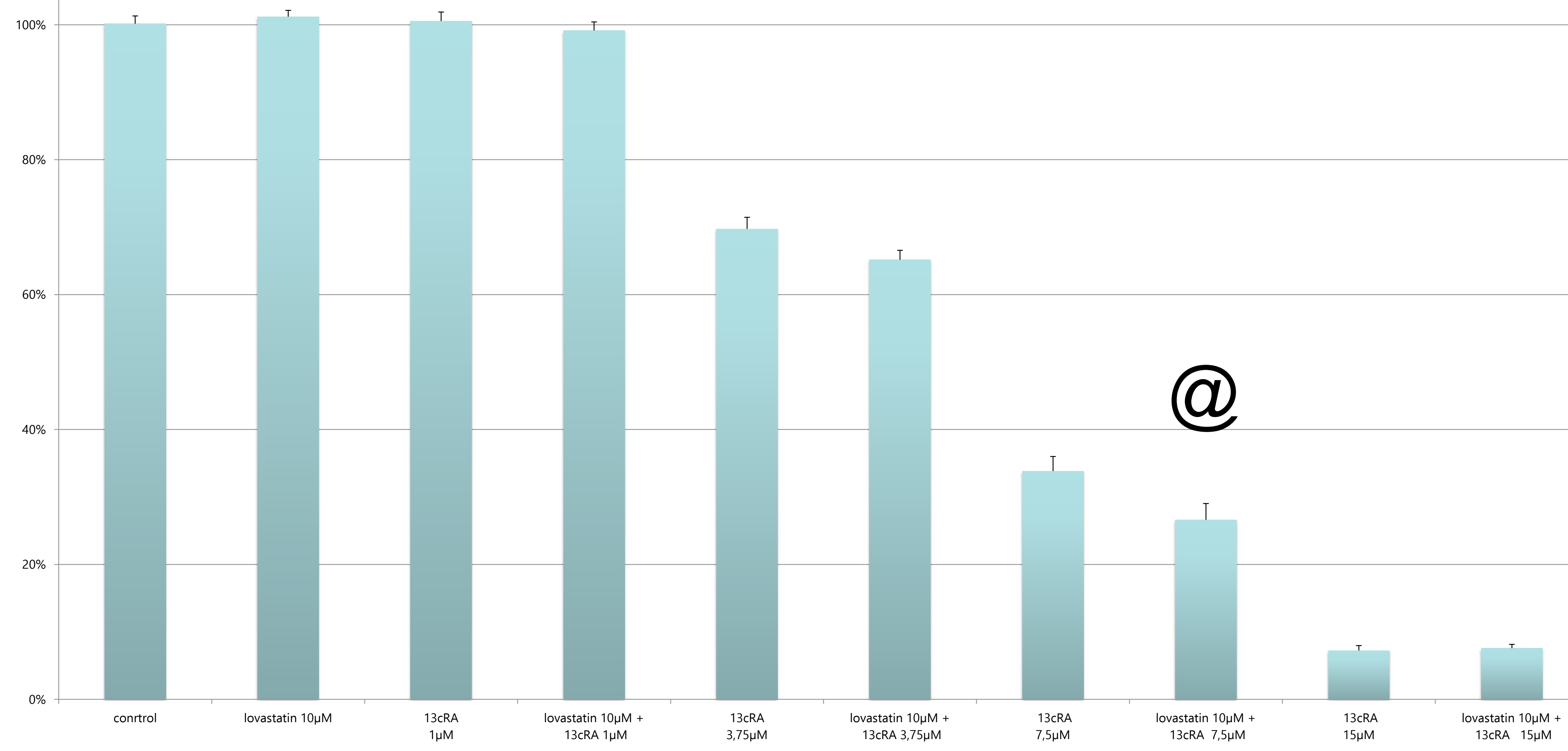

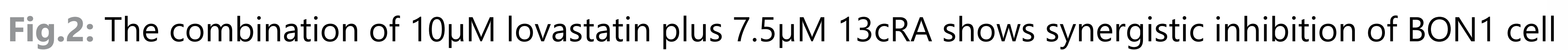
viability ( $\mathrm{p} \leq 0.05)(@)$

\section{Conclusion}

-Synergistic effect of 13cRA plus lovastatin in BON1 cells at clinically relevant doses, associated with enhanced EGFR inhibition

- Consistent with our in vivo data in a phaeochromocytoma allograft model showing slowest tumour growth and lowest microvessel density after 13cRA/lovastatin-treatment -Potentially novel effective drug combination

Fig.4 Bon1 $\mathrm{H} 727$

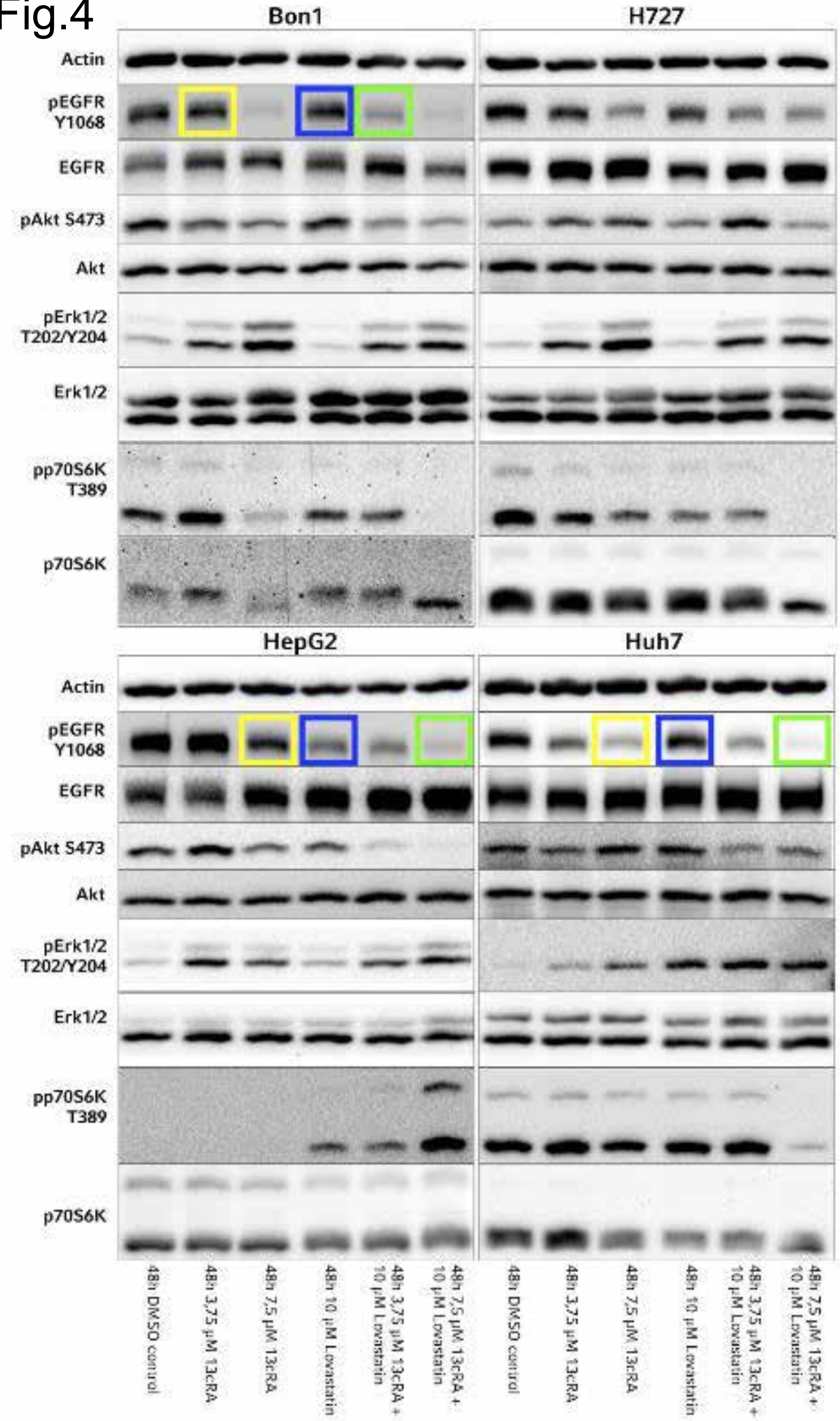

Fig.4: Exemplary western blots of each cell line tested: In BON1, HepG2 and Huh7 cells, combination-treatment (green) decreases pEGFR by more than $50 \%$ relative to each drug separately (yellow/blue), and by more than $80 \%$ relative to the untreated control. This effect is less pronounced in $\mathrm{H} 727$ cells. 